\title{
A case of Gonococcal septic arthritis involving left shoulder joint
}

\author{
N.H. Kumarasinghe ${ }^{1}$, H.Perera ${ }^{2}$
}

\begin{abstract}
In the modern era where antibiotics are freely available, gonococcal septic arthritis is a rarity. Here we report a probable case of localized gonococcal septic arthritis involving the shoulder joint. There are no such cases reported from Sri Lanka up to now.
\end{abstract}

Key Words: Gonococcal Septic Arthritis, shoulder joint, disseminated gonococcal infection

Authors: ${ }^{D}$ Dr N.H.Kumarasinghe (MBBS,PgD Ven), Registrar in Venereology, National STD/AIDS Control Pgramme, Colombo, Sri Lanka. ${ }^{2}$ Dr H. Perera, (MBBS, PgDVen, MD); Consultant Venereologist, National STD/AIDS Control Programme, Colombo, Sri Lanka. Email:pererahimali.perera@gmail.com

Copyright

Corresponding author: ${ }^{1 D}$ N.H.Kumarasinghe, Email:nadeera_kumarasinghe@yahoo.com

Conflict of interest: None

Financial support: None, self-financing

Originality: This is an original work there has been no previous publications.

Submitted on: 29.11.2018; Accepted on 20.12.2018

\section{Case Report}

Introduction

Gonorrhoea is a sexually transmitted Infection(STI) which has a profound impact on sexual and reproductive health. Worldwide, out of the estimated 357 million cases of new STIs, contribution of Neisseria gonorrheae infections was 78 million. (1) During 2017, 427 new gonorrhea cases were reported from Sri Lanka.(2) Generally, gonorrhea infections resolve without complications in males. Gonococcal arthritis is a rare phenomenon with only a few reported cases globally.(3) There are no reported cases of gonococcal septic arthritis from Sri Lanka.

\section{Case History}

A 40-year-old married male with fever, pain and swelling of left shoulder joint for 3 weeks presented to the National Hospital of Sri Lanka. As there was also a yellowish, offensive urethral discharge he was referred to the central STD clinic for further management. He had mild dysuria. He had no history of diabetes mellitus, rheumatoid arthritis, osteoarthritis, gout, renal stones or psoriasis. He strongly denied extra marital sexual exposures or casual sex

On examination left shoulder joint was swollen and tender. Left shoulder joint movements were severely restricted with abduction possible only up to 30 degrees from the vertical plane. Other movements were also restricted to around 30 degrees. All other joints were clinically normal. There were no skin rashes or lesions. Palms and soles were normal. There were no crusty plaques, vesicopustular waxy lesions suggestive of keratodermablenorrhagica.

Gram stain of the urethral discharge showed intracellular and extracellular gram-negative diplococci. Gonorrhoea culture was positive. Venereal Disease Research Laboratory (VDRL) and HIV Enzyme Linked Immunosorbent Assay (ELISA) were negative.

The patient was treated with intravenous ceftriaxone $1 \mathrm{~g}$ daily for 3 days, followed by oral cefixime $400 \mathrm{mg}$ and doxycycline 100mg twice daily for 7 days. 


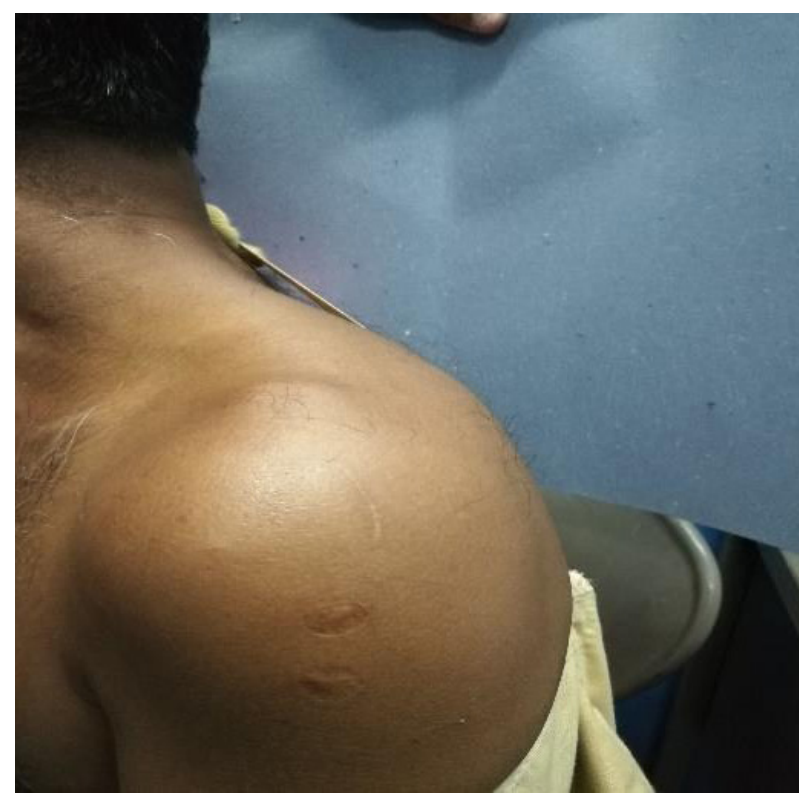

Figure 1: Lateral view of left shoulder joint

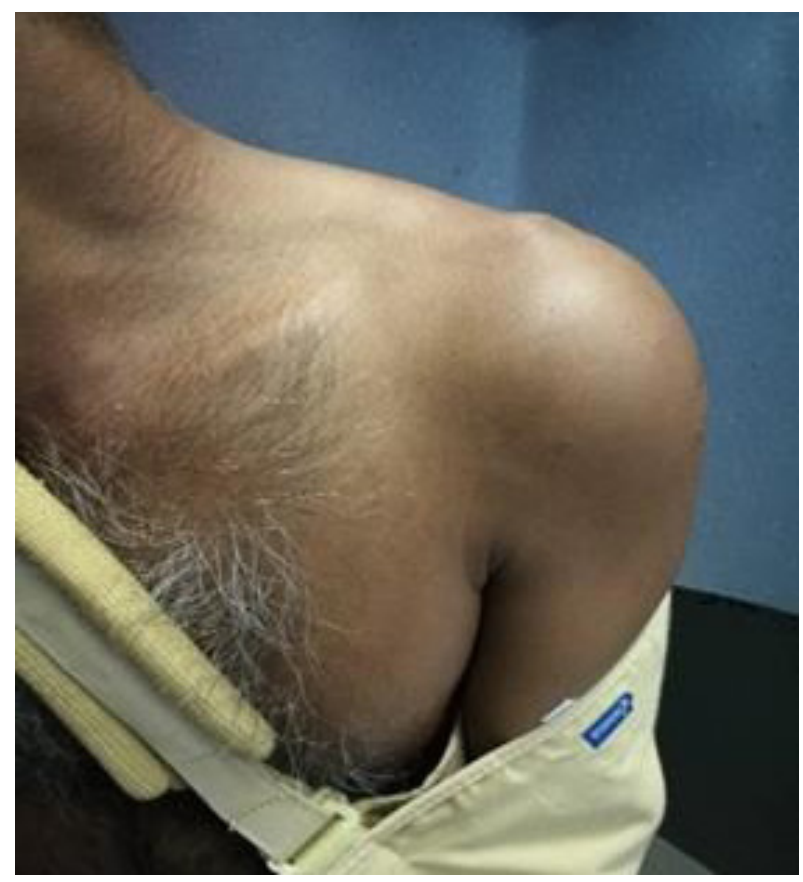

Figure 2: Antero- posterior view of left shoulder joint

He responded well to antibiotics where intracellular and extracellular Gram-negative diplococci were absent in the repeated urethral smear and culture after 3 days of treatment.

\section{Discussion}

Approximately $0.5 \%$ to $3 \%$ of patients infected with Neisseria gonorrhoeae develop Disseminated gonococcal infection (DGI).(3) Gonococcal arthritis can occur in otherwise healthy individuals. It may occur at any age.

Disseminated Gonococcal infection consists of two major clinical syndromes: "the arthritis-dermatitis syndrome and localized purulent arthritis without associated skin lesions". Some patients can present with overlapping clinical feature of two syndromes. Localized septic arthritis can present as a monoarthritis or asymmetric oligo-or polyarthritis, with pain and swelling of one or more joints. Most patients do not have systemic symptoms. Commonly affected joints are knees, ankles, wrist, and elbow. Shoulder joint involvement as in our patient is not a common presentation, but few cases have been reported in medical literature.(3)

Confirmation of Gonococcal infection is through isolation of Neisseria gonorrhoeae from the infected site. DGI or gonococcal arthritis is diagnosed by identification of gram-negative intracellular diplococci, in a specimen taken from a non-mucosal site such as blood, synovial fluid, or a skin lesion. As microbiological tests are not always positive in DGI, in many cases diagnosis is made clinically.

Standard treatment gonococcal arthritis is intravenous or intramuscular ceftriaxone $1 \mathrm{~g}$ once daily for one to two days, followed by cefixime 400 $\mathrm{mg}$ orally twice daily for 1 week. Doxycycline $100 \mathrm{mg}$ twice daily for 7 days is added to cover potential coinfection with Chlamydia trachomatis.(4)

\section{References}

1. World Health Organization. Sexually transmitted and reproductive tract infections. . 2017.

2. National STD/AIDS Control Programme, Annual Report. 2017.

3. Arthritis, Gonococcal. Li, Raymund and Hatcher1., Jason D. October 25, 2018, Treasure Island (FL): StatPearls Publishing; 2018 Jan-. Available from: https://www.ncbi. nlm.nih.gov/books/NBK470439/.

4. Sexually transmitted infections management guideline. Sri Lanka : National STD/AIDS Control Programme, 2009. 